\title{
BÚSQUEDA DE INFECCIÓN POR HEPATITIS B EN FAMILIARES DE PORTADORES CRÓNICOS DE LA PROVINCIA DE HUANTA, AYACUCHO -PERÚ
}

\author{
César Cabezas ${ }^{1,2, a}$, Johanna Balbuena-Torres ${ }^{2, b}$, Luis Javier Crispín Huamaní i,3,c, \\ Cristhian Landa Huaman ${ }^{1,3, c}$, Jeniffer Rios-Rojas ${ }^{1,3, c}$, Andrea Sucari Idrogo ${ }^{1, c}$, \\ Andrea Anampa-Guzmán ${ }^{1,4, c}$
}

\begin{abstract}
RESUMEN
Un plan de eliminación del virus de hepatitis B (HBV) es factible porque la inmunización ha tenido buen impacto, tal como ha sucedido en la provincia de Huanta en Perú. El objetivo de nuestro estudio fue determinar la frecuencia de la infección por HBV en familiares de portadores del antígeno de superficie del virus de la hepatitis B (HBsAg). Este estudio transversal incluyó a 39 familiares de portadores crónicos, identificados en el Hospital de Apoyo de Huanta. Se recolectaron datos sociodemográficos y muestras de sangre. La frecuencia total de infección por HBV fue de 10,3 \% y la mayoría correspondía a infección crónica $(7,7 \%)$. Una tercera parte tenía antecedentes de infección por HBV. Los miembros de la familia con infección por HBV fueron mayormente adultos alcohólicos y no vacunados. En conclusión, encontramos una alta frecuencia de HBV en familiares de portadores de HBsAg, esta estrategia ayudaría a identificar portadores crónicos que pueden ser tratados y contribuir a un plan de eliminación de HBV.
\end{abstract}

Palabras claves: Hepatitis B; Prevalencia; Transmisión familiar; Eliminación (fuente: DeCS BIREME).

\section{SEARCH OF HEPATITIS-B INFECTION IN RELATIVES OF CHRONIC CARRIERS IN THE PROVINCE OF HUANTA, AYACUCHO, PERU}

\begin{abstract}
S
A plan of elimination of the virus of B hepatitis (HBV) is feasible because the immunization has had good impact, as it has been documented in the province of Huanta in Peru. The objective of our study was to determine the frequency of the infection by HBV in relatives of carriers of the surface antigen of the virus of hepatitis B (HBsAg). This cross-sectional study included 39 relatives of chronic carriers, identified at Hospital de Apoyo de Huanta. Sociodemographic data and blood samples were collected. The total frequency of infection by HBV was $10.3 \%$, and the majority corresponded to chronic infection $(7.7 \%)$. One third had a history of infection by HBV. The family members with HBV infection were mainly adult alcoholics who had not been vaccinated. In conclusion, we found a high frequency of HBV in relatives of carriers of HBsAg. This strategy would help identify chronic carriers that can be treated and to contribute to a plan for the elimination of HBV.
\end{abstract}

Keywords: Hepatitis B, Prevalence; Family transmission, Elimination (source: MeSH NLM).

\section{INTRODUCCIÓN}

La infección por el virus de la hepatitis B (HBV) constituye la décima causa de muerte en el mundo debido a su forma de presentación, provocando casi 600 mil muertes al año ${ }^{(1-4)}$. Las complicaciones graves como insuficiencia hepática y hepatocarcinoma se presentan en el $15 \%$ a $25 \%$ del total de pacientes con infección crónica por HBV ${ }^{(5)}$. A pesar de que existen programas de vacunación, la infección por HBV está presente en toda Latinoamérica. En Perú, las personas no vacunadas tienen la mayor mortalidad por HBV que en todo el continente americano ${ }^{(6)}$.

La coinfección con el virus de la hepatitis D (HDV) está relacionado con un mayor riesgo de enfermedad fulminante o crónica ${ }^{(6,7)}$. Sin embargo, luego del inicio del programa de

\footnotetext{
Facultad de Medicina Humana, Universidad Nacional Mayor de San Marcos. Lima, Perú

Centro Nacional de Salud Pública, Instituto Nacional de Salud. Lima, Perú.

Asociación para el Desarrollo de la Investigación Estudiantil en Ciencias de la Salud, Universidad Nacional Mayor de San Marcos. Lima, Perú

Sociedad Científica de San Fernando, Facultad de Medicina Humana, Universidad Nacional Mayor de San Marcos. Lima, Perú

Médico infectólogo tropicalista; ${ }^{\mathrm{b}}$ bióloga; ${ }^{\mathrm{c}}$ estudiante de Medicina

Recibido: 25/07/2018 Aprobado: 28/11/2018 En línea: 21/12/2018
}

Citar como: Cabezas C, Balbuena-Torres J, Crispin Huamani LJ, Landa Huamán C, Rios-Rojas J, Sucari Idrogo A, Anampa-Guzmán A. Búsqueda de infección por hepatitis B en familiares de portadores crónicos de la provincia de Huanta, Ayacucho-Perú. Rev Peru Med Exp Salud Publica. 2018;35(4):647-51. doi:10.17843/ rpmesp.2018.354.3833. 
vacunación en el Perú, se produjo la reducción de las tasas de mortalidad por enfermedades hepáticas relacionadas con la hepatitis $B$, en particular cirrosis, hepatocarcinoma y hepatitis fulminante en niños menores de 15 años ${ }^{(8)}$. A pesar de que la tasa de nuevas infecciones se han reducido después de la implementación del programa de vacunación ${ }^{(9)}$, el legado de infección se mantiene y deja al individuo infectado crónicamente con un riesgo muy alto de desarrollar enfermedades hepáticas.

En Perú, a pesar de ser un país con una importante proporción de personas infectada por HBV, sólo se ha realizado un estudio sobre trasmisión intrafamiliar, no encontrándose riesgo de transmisión pero si de coinfección con HDV (10). Huanta fue una ciudad reconocida por sus antecedentes de tener una alta prevalencia de hepatitis $A$, $B$ y $D^{(11)}$, sin embargo, luego del impacto de la inmunización contra HBV realizada en esta ciudad y después de universalizar la vacunación en todo el país, actualmente es posible implementar un plan de eliminación de esta infección.

La vacunación es realizada por el estado peruano de manera gratuita. Sin embargo, sería importante intensificar las estrategias de búsqueda e identificación de casos a través de la inmunización activa contra la HBV e implementar un programa de intervención a los portadores crónicos de la infección ${ }^{(12)}$. Actualmente, no existe una estrategia para la identificación de casos, la mayoría son reconocidas de manera incidental.

En este contexto, realizamos un estudio con el objetivo de determinar la frecuencia de la infección por HBV en los familiares de portadores crónicos de HBsAg. Corresponde a un estudio piloto basado en la búsqueda de portadores crónicos de HBV, para tratarlos y junto con las medidas de vacunación, ya establecidas por el Ministerio de Salud, contribuir con la eliminación de la HBV en esta zona. Este sería el primer intento de la eliminación de la HBV, iniciativa que serviría de modelo para otras regiones del país.

\section{EL ESTUDIO}

Se realizó un estudio descriptivo en los contactos familiares de pacientes que son portadores crónicos del antígeno de superficie del virus de la hepatitis $B$ (HBsAg) (caso índice) en la provincia de Huanta, departamento de Ayacucho en Perú durante el 2016. Al tratarse de un estudio piloto, los sujetos fueron incluidos por conveniencia. Los portadores crónicos fueron identificados en los registros del Hospital de Apoyo de Huanta.

Se definió como contacto familiar a las personas que residían o habían residido con el caso índice en la misma vivienda por un periodo no menor de seis meses y que se encontraban en Huanta durante los tres días de recolección de datos. En caso de ser menor de cinco años, sólo se incluyeron a aquellos cuya madre era portadora crónica.

\section{MENSAJES CLAVE}

Motivación para realizar el estudio. En áreas endémicas de infección por hepatitis viral (HBV) donde el estado de portador usualmente es asintomático, ameritaba contar con una estrategia para la identificación de estos casos.

Principales hallazgos. Se encontró que uno de cada diez familiares de portadores del antígeno de superficie (HBsAg) tiene infección por el HBV.

Implicancias. Esta estrategia contribuirá a identificar y tratar a portadores crónicos de HBV complementando las acciones de inmunización de la población infantil en el contexto de un programa de eliminación de la HBV.

Una semana antes de la recolección de datos, se contactó vía telefónica con los participantes a fin de citarlos en el Hospital de Apoyo de Huanta para la obtención de muestras sanguíneas. Se les explicó el objetivo del estudio y se obtuvo el consentimiento informado. En los menores, se obtuvo el asentimiento informado, además del consentimiento y permiso de sus padres.

Se aplicó un cuestionario donde se consignaron datos sociodemográficos (edad, sexo, procedencia, ocupación, estado civil, grado de instrucción); estado de vacunación; relación con el caso índice y factores de riesgo (número de parejas sexuales, cirugías y procedimientos médicos, tatuajes, alcoholismo y vacunación). Los encuestadores fueron estudiantes de medicina previamente entrenados. Los cuestionarios fueron aplicados de forma anónima y confidencial, antes de la obtención de la muestra de sangre.

A cada participante se le tomó una muestra de $5 \mathrm{ml}$ de sangre venosa en dos tubos, uno con anticoagulante EDTA y otro sin anticoagulante. Las muestras fueron transportadas al laboratorio del Hospital de Apoyo de Huanta a $-4{ }^{\circ} \mathrm{C}$ para la separación del suero mediante centrifugación.

Las muestras séricas fueron almacenadas a $-20^{\circ} \mathrm{C}$ hasta su envío en cadena de frío $\left(4^{\circ} \mathrm{C}\right.$ a $\left.8{ }^{\circ} \mathrm{C}\right)$ de acuerdo con las normas de bioseguridad y de transporte de muestras biológicas de categoría $B{ }^{(14)}$. Se enviaron al Instituto Nacional de Salud (INS), donde se realizó el procesamiento serológico mediante la prueba ELISA para los marcadores: $\mathrm{HBsAg}$, anti-HBc y anti-HBs. Las muestras con resultado positivo para el $\mathrm{HBsAg}$, adicionalmente, fueron evaluadas para el HBeAg, anti-HBc lgM, anti-HDV-lgM y anti-HDV-lgG.

La prueba ELISA se realizó en lavador y lector de ELISA marca Robonik, con reactivos de marca Wantai, (Beijing wantai Biological Pharmacy) ${ }^{(15)}$ siguiendo las indicaciones del fabricante. La cuantificación del ADN-HBV en el plasma de los pacientes con infección crónica por VHB se realizó en el extractor Cobas Ampliprep y el termociclador Cobas TaqMan 48, mediante la reacción en cadena de la polimerasa 
en tiempo real (qPCR) utilizando los kits COBAS AmpliPrep /COBAS TaqMan VHB Test, versión 2.0 (Roche Molecular Diagnostics, Branchburg, Nueva Jersey), cuyo límite inferior de detección es de $20 \mathrm{Ul} / \mathrm{mL}^{(16)}$. El laboratorio cuenta con un sistema de gestión de calidad de los procedimientos y los resultados.

La infección crónica por HBV se definió como la presencia de HBsAg y anti-HBc, y la ausencia del Anti-HBc-lgM. La infección pasada se determinó por la presencia de anti$\mathrm{HBc}$ y del anti-HBs. La infección aguda se definió como la presencia de HBsAg y del Anti HBc-lgM. Los susceptibles fueron negativos a HBsAg, anti-HBc y anti-HBs. Los sujetos inmunizados tuvieron únicamente resultado positivo para el anti-HBs.

Los datos fueron incorporados en una base en Excel 2013, y analizados con el programa estadístico Stata versión 13. Una persona se negó a llenar la encuesta, pero aceptó el examen serológico de HBV. Finalmente, se realizó un análisis descriptivo para todas las variables de estudio. Las variables categóricas se expresaron a través de frecuencias y porcentajes y las numéricas a través de medias y rangos.

La participación en la investigación fue libre y voluntaria como se estipula en el consentimiento informado. El protocolo del estudio fue aprobado por el comité de Ética del Instituto de Medicina Tropical de la Universidad Nacional Mayor de San Marcos.

\section{RESULTADOS}

Se analizaron las características de 39 familiares de 15 casos índices de infección crónica por HBV. En la Tabla 1 se muestra las características sociodemográficas de los

Tabla 1. Frecuencia de infección por el virus de la hepatitis $B$ en contactos familiares de portadores crónicos según características sociodemográficas

\begin{tabular}{lcc}
\hline Característica & $\begin{array}{c}\text { Sin infección } \\
\text { por HBV } \\
\mathbf{n}(\%)\end{array}$ & $\begin{array}{c}\text { Con infección } \\
\text { por HBV } \\
\mathbf{n}(\%)\end{array}$ \\
\hline Edad* $^{*}$ & $23,7(18,7)$ & $45(9,8)$ \\
\hline Hombres & $15(93,8)$ & $1(6,2)$ \\
\hline Mujeres & $20(87,0)$ & $3(13,0)$ \\
\hline Vive en zona endémica & $23(88,5)$ & $3(11,5)$ \\
\hline Personal de salud & $3(100,0)$ & $0(0,0)$ \\
\hline Soltero & $18(94,7)$ & $1(5,3)$ \\
\hline Analfabeto & $26(89,7)$ & $3(10,3)$ \\
\hline Total & $35(89,7)$ & $4(10,3)$ \\
\hline
\end{tabular}

*Media (desviación estandar)

HBV: virus de la hepatitis B contactos familiares, donde se aprecia que la edad promedio es mayor en las personas infectadas por HBV.

La frecuencia global de infección por HBV fue de 10,3\% y la mayoría correspondía a infección crónica $(7,7 \%)$. Asimismo, una tercera parte había tenido antecedente de infección por HBV (Tabla 2). Todos los pacientes con infección crónica por HBV $(n=3)$ eran mayores de 23 años. Todos ellos fueron $\mathrm{HBeAg}$ negativo y negativo para coinfección con hepatitis $D$. Los valores de carga viral resultaron no detectables en un participante y entre 20$1000 \mathrm{UI} / \mathrm{ml}$ en los otros dos participantes.

En la Tabla 3 se muestra los factores de riesgo para infección por HBV en los contactos familiares de los portadores crónicos de HBsAg. La mayoría de los familiares que tenían la infección por HBV habían sido sometidos a procedimientos dentales, padecían de alcoholismo y habían sido vacunados previamente contra HBV. Ninguno de los miembros de la familia estaba infectados con el HDV.

\section{DISCUSIÓN}

En países con gran endemicidad, la transmisión intrafamiliar es unavía común de contagio de HBV ${ }^{(17,18)}$. En Latinoamérica, la prevalencia de infección por HBV se encuentra entre baja e intermedia, con variaciones que dependen del nicho ecológico-social en cada país ${ }^{(19)}$. Un estudio brasileño proporcionó fuerte evidencia de transmisión intrafamiliar en familias con más de un miembro con HBsAg positivo y entre los que habitualmente comparten materiales de aseo como cepillos de dientes ${ }^{(20)}$. El Perú tiene intervalos altos e intermedios de infección (20) con tasas de portadores de HBsAg entre $0,8 \%$ a 3,5 \%. Regiones como Loreto y Abancay constituyen zonas con una prevalencia $\mathrm{HBsAg}$ mayor al $10 \%$. La frecuencia relativa de infección por HBV entre los familiares de portadores crónicos de HBsAg en Huanta fue de $10 \%$.

La infección por HBV produce hasta $3 \%$ de la mortalidad total, aunque en áreas hiperendémicas como Huanta (Ayacucho) y Abancay (Apurímac), esta llegaría al $7 \%$. Hasta el $40 \%$ de peruanos con hepatocarcinoma provienen

Tabla 2. Frecuencia de infección por el virus de la hepatitis $B$ en los contactos familiares de portadores crónicos

\begin{tabular}{lr}
\hline Característica & n (\%) \\
\hline Infección global por HBV & $4(10,3)$ \\
\hline Infección crónica por HBV & $3(7,7)$ \\
Infección aguda por HBV & $1(2,6)$ \\
Sin infección por HBV & $35(89,7)$ \\
Infección pasada por HBV & $13(33,3)$ \\
\hline
\end{tabular}

HBV: virus de la hepatitis $B$ 
Tabla 3. Factores de riesgo de infección por el virus de la hepatitis B en familiares de portadores crónicos*

\begin{tabular}{lcc}
\hline Factores $^{*}$ & $\begin{array}{c}\text { Sin infección } \\
\text { por HBV } \\
\mathbf{n}(\%)\end{array}$ & $\begin{array}{c}\text { Con } \\
\text { infección } \\
\text { por HBV } \\
\mathbf{n}(\%)\end{array}$ \\
\hline $\begin{array}{l}\text { Número de parejas } \\
\text { sexuales }\end{array}$ & $0,6(1,07)$ & $1,0(1,0)$ \\
\hline Cirugía previa $^{\dagger}$ & $6(85,7)$ & $1(14,3)$ \\
\hline Procedimientos dentales & $21(87,5)$ & $3(12,5)$ \\
\hline Tatuajes & $1(100,0)$ & $0(0,0)$ \\
\hline Alcoholismo & $16(88,9)$ & $2(11,1)$ \\
\hline Vacunación contra HBV & $20(90,9)$ & $2(9,1)$ \\
\hline
\end{tabular}

* Solo 38 contactos familiares respondieron la encuesta

† Media (Desviación estándar)

HBV: virus de la hepatitis B

de zonas endémicas de hepatitis B (Madre de Dios, Abancay y Huanta) ${ }^{(20)}$. En nuestro estudio sólo había un menor de edad con infección por HBV, esto podría deberse a la vacunación iniciada desde 1994.

La vacuna para HBV es considerada el principal método de prevención y ha tenido buenos resultados en la reducción de las tasas de infección crónica en países en las que más del $8 \%$ de su población infantil padecía de esta condición ${ }^{(2)}$. Un estudio realizado en Irán muestra una disminución significativa de la tasa de infección por VHB, tras el uso de la vacuna, en niños de 2 a 14 años, en comparación con adultos de 15 a 69 años ${ }^{(22)}$.

Cuando se evaluaron otras características, se observó que el número de parejas sexuales es relativamente mayor en los sujetos no infectados; no obstante, la transmisión horizontal que ocurre en la infancia es más predominante que la transmisión sexual (17). En el estudio de Mansour-Ghanaei et al., en 415 familiares de portadores crónicos de HBV, no se encontró asociación con antecedentes de procedimientos dentales, tatuajes, accidentes punzocortantes o uso de drogas endovenosas ${ }^{(21)}$

Este es el primer piloto que estudia la infección en contactos domiciliarios que viven con casos índice en Perú. Algunas limitaciones de nuestro estudio son: primero, al tratarse de un piloto se contó con un número relativamente bajo de participantes; segundo, la población fue heterogénea al haber incluido adultos y menores de edad, tercero, no se realizó ningún seguimiento a los participantes y finalmente, los participantes proceden de un área hiperendémica lo que podría sobreestimar los resultados. Se sugiere realizar estudios de mayor escala, sistematizando la información dentro del plan de eliminación de HBV y que además refuerce la inmunización infantil activa.

Concluimos que, uno de cada diez familiares de portadores de HBsAg tiene infección por HBV. Futuros estudios deberían de analizar el costo beneficio de esta estrategia para identificar portadores crónicos susceptibles de ser tratados y contribuir en un plan de eliminación de la HBV, que tiene además como acción principal la inmunización de la población infantil.

Contribuciones de autoría: $\mathrm{CC}$ ha participado en la concepción y diseño del artículo. CC, JBT, LCH, KLH, KRR, ASI y AAG contribuyeron con recolección de la información y la redacción del artículo. CC participó en la revisión crítica del artículo. AAG contribuyó con el análisis e interpretación de datos y asesoría estadística. CC y JBT contribuyeron con aporte de pacientes o material de estudio y obtención de financiamiento y asesoría técnica o administrativa. CC, JBT, LCH, KLH, KRR, ASI y AAG aprobaron la versión final del artículo.

Financiamiento: El Instituto Nacional de Salud financió parcialmente el presente estudio.

Conflictos de interés: Ninguno.

\section{REFERENCIAS BIBLIOGRÁFICAS}

1. Salkic NN, Zildzic M, Muminhodzic K, Pavlovic-Calic N, Zerem E, Ahmetagic $S$, et al. Intrafamilial transmission of hepatitis B in Tuzla region of Bosnia and Herzegovina. Eur J Gastroenterol Hepatol. 2007;19(2):113-8.

2. World Health Organization. Hepatitis B [Internet]. WHO. [citado el 22 de mayo de 2018]. Disponible en: http://www. who.int/mediacentre/factsheets/fs204_ Jul2014/en/

3. Goldstein ST,Zhou F, HadlerSC, Bell BP, Mast EE, Margolis HS. A mathematical model to estimate global hepatitis B disease burden and vaccination impact. Int J Epidemiol. 2005;34(6):1329-39.
4. Ganem D, Prince AM. Hepatitis B virus infection--natural history and clinical consequences. N Engl J Med. 2004;350(11):1118-29.

5. Centers for Disease Control. Hepatitis Bgeneral information Atlanta [Internet]: Department of Health \& Human Services. Centers for Disease Control and Prevention; 2010 [citado el $30 \mathrm{de}$ octubre de 2018]. Disponible en: http:// www.cdc.gov/hepatitis/hbv/pdfs/ hepbgeneralfactsheet.pdf

6. Alvarado-Mora MV, Pinho JRR. Epidemiological update of hepatitis $\mathrm{B}, \mathrm{C}$ and delta in Latin America. Antivir Ther. 2013;18(3 Pt B):429-33.
7. Parkin DM. Global cancer statistics in the year 2000. Lancet Oncol. 2001;2(9):533-43.

8. Ramírez-Soto MC, Ortega-Cáceres G, Cabezas C. Trends in mortality burden of hepatocellular carcinoma, cirrhosis, and fulminant hepatitis before and after roll-out of the first pilot vaccination program against hepatitis B in Peru: An analysis of death certificate data. Vaccine. 2017;35(31):3808-12.

9. Méndez M, Arce M, Kruger H, Sánchez S. Prevalence of serologic markers of viral hepatitis in various population groups in Peru. Boletin Oficina Sanit Panam Pan Am Sanit Bur. 1989;106(2):127-38. 
10. Segovia MG, Galván $\mathrm{K}$, García $\mathrm{AV}$, Huamaní L, Gotuzzo HE. Prevalencia de marcadores serológicos para hepatitis B y delta e infección intrafamiliar en el valle del río Pampas, Perú. Rev Peru Med Exp Salud Publica. 2002;19(2):57-62.

11. Cabezas C, Gotuzzo E, Escamilla J, Phillips I. Prevalence of serological markers of viral hepatitis A, B and delta in apparently healthy schoolchildren of Huanta, Peru. Rev Gastroenterol Peru.1994;14(2):123-34.

12. Cabezas Sánchez C. Situación y control de la hepatitis B y Delta en el Perú. Acta Médica Peru. 2008;25(2):96-112

13. World Health Organization. Guidance on regulations for the Transport of Infectious Substances 2015-2016. [Internet]. [Consultado 27 oct 2018]. Disponible en: http://apps.who.int/ iris/bitstream/handle/10665/149288/ WHO_HSE_GCR_2015.2_eng.pdf;jsessionid=8D3199B66B5491FE6CC67518B68E58AF sequence $=1$

14. Beijing wantai Biological Pharmacy. Hepatitis B [Internet]. [Consultado 27 oct 2018]. Disponible en: http://www.ystwt. $\mathrm{cn} /$ hepatitis-b/
15. Roche Molecular Systems, Inc. COBAS AmpliPrep/COBAS ${ }^{\curvearrowleft}$ TaqMan $^{\circ} \mathrm{HBV}$ Test. [Internet]. [Consultado 27 oct 2018]. Disponible en: https://pim-eservices.roche.com/eLD_SF/pe/es/Documents/GetDocument?documentId=8f4 ef27d-6b6b-e511-f1bd-00215a9b3428

16. Morales J, Fuentes-Rivera J, Delgado-Silva C, Matta-Solís H. Viral Hepatitis Infection Markers Among Blood Donor in a National Hospital of Metropolitan Lima. Rev Peru Med Exp Salud Publica. 2017;34(3):466-71.

17. Tanaka J. Hepatitis B epidemiology in Latin America. Vaccine. 2000;18 Suppl $1:$ S17-9.

18. Lobato C, Tavares-Neto J, Rios-Leite M, Trepo C, Vitvitski L, Parvaz P, et al. Intrafamilial prevalence of hepatitis B virus in Western Brazilian Amazon region: epidemiologic and biomolecular study.J Gastroenterol Hepatol. 2006;21(5):863-8.

19. Cabezas C. Hepatitis viral B y delta en el Perú: Epidemiología y bases para su control. Rev Peru Med Exp Salud Publica. 2007;24(4):378-97.
20. Cabezas C, Anaya E, Bartalesi F, Sánchez J. Transmisión Horizontal de Hepatitis Viral B en población desplazada de un área Hiperendémica a sus contactos en un área de baja endemicidad del Perú. Rev Gastroenterol Peru. 1997;17(2):128-34.

21. Mansour-Ghanaei F, Joukar F, Yaseri M, Soati F, Atrkar-Roushan Z. Intrafamilial spread of hepatitis B virus in Guilan Province-North of Iran. Int $\mathrm{J}$ Mol Epidemiol Genet. 2013;4(4):250-7.

22. Zali MR, Mohammad K, Noorbala AA, Noorimayer B, Shahraz S, Sahraz S. Rate of hepatitis B seropositivity following mass vaccination in the Islamic Republic of Iran. East Mediterr Health J Rev Sante Mediterr Orient Al-Majallah Al-Sihhiyah Li-Sharq Al-Mutawassit. 2005;11(1-2):62-7.

Correspondencia: Andrea Carolina Anampa Guzmán

Dirección: Av. Villaverde 308 - Urb. San Amadeo de Garagay. San Martin de Porres. Lima, Perú

Teléfono: (511) 998455065

Correo electrónico:andrea.anampa@outlook.com.pe

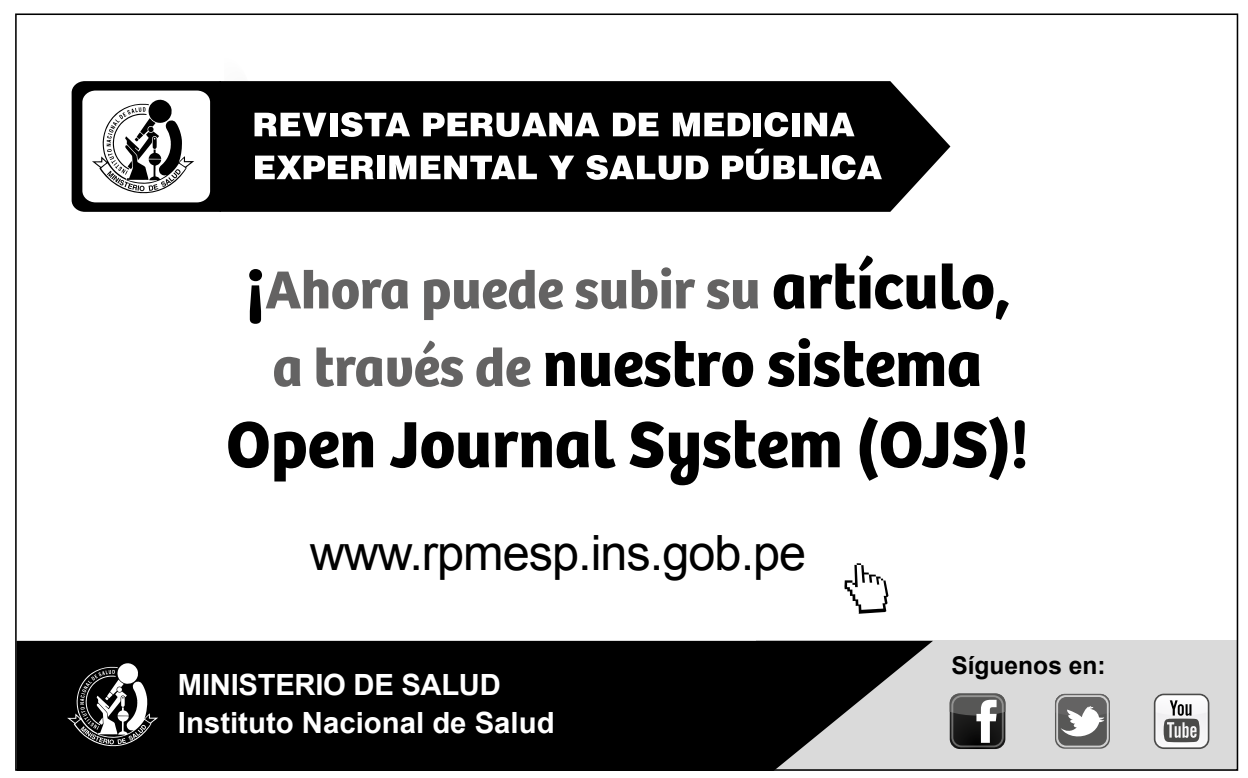

